\title{
Multi-View Planning for Simultaneous Coverage and Accuracy Optimisation
}

\author{
Christoph Munkelt ${ }^{1}$ \\ christoph.munkelt@iof.fraunhofer.de \\ Andreas Breitbarth ${ }^{1}$ \\ andreas.breitbarth@iof.fraunhofer.de \\ Gunther Notni ${ }^{1}$ \\ gunther.notni@iof.fraunhofer.de \\ Joachim Denzler ${ }^{2}$ \\ joachim.denzler@uni-jena.de
}

${ }^{1}$ Fraunhofer Institute for Applied Optics and Precision Engineering IOF Jena, Germany

${ }^{2}$ Friedrich Schiller University of Jena, Chair for Computer Vision Jena, Germany

\begin{abstract}
View planning for three-dimensional (3D) reconstruction and inspection solves the problem of finding an efficient sequence of views allowing complete and high quality reconstruction of complex objects. To fulfil this task, view planning methods need to deal with sensor limitations and satisfy predefined goals. Our objective is to jointly evaluate accuracy requirements and coverage during planning, to optimise the reconstruction procedure, as well as to explicitly take configuration space constraints into account.

We present a novel model-based approach, which at the same time optimises accuracy and coverage based on an existing model (e.g. CAD or preview scan). For accuracy optimisation we extended the statistical $E$-criterion to model directional uncertainty. Coverage is maximised while taking configuration space and sensor characteristics into account. We validate our approach through experimental evaluation using the Next-bestview (NBV) benchmark framework and a robot mounted stereo fringe projection sensor.
\end{abstract}

\section{Introduction}

Multi-View Planning (MVP) is a key problem for high fidelity 3D inspection and object reconstruction. Given a CAD model - or coarse 3D scan, or time of flight (TOF) 3D scan - of the object, MVP determines a set of views. One important aspect is to find a minimal number of views that allow for complete object reconstruction. Additionally, for high fidelity scanning aiming at "scanning precision of a few tens of microns at a density of many samples per square millimeters" [四], an upper bound for reconstruction error is crucial.

In this paper we present a novel model-based MVP approach, which models measurement uncertainty additionally to coverage. First, Scott's fundamental 3M approach [四] is adapted to deliver higher coverage for complex measurement objects in the presence of noteworthy configuration space (CS) restrictions (Section 3.1). We then introduce an uncertainty estimation scheme. Using this prediction, we extend the $E$-criterion [ $\square$ ] to determine viewpoints that minimise overall reconstruction uncertainty. Our paper's contribution is the 
accuracy optimising MVP approach using the extended E-criterion, simultaneously taking uncertainty and coverage into account (Sec. 3.2). Finally, we evaluate different planning methods using the benchmark object and scheme from [] (Sec. 4).

\section{Literature Review}

For an in-depth view planning survey, see Scott et al. []]. For brevity, we focus on modelbased methods. While Tarbox' and Gottschlich's [] approach was limited to viewpoint candidate creation on a view sphere around the object, they introduced the concept of complete visibility analysis over the object's surface. Using the resulting visibility matrix, they capitalised on the isomorphism of the view planning problem to the set covering problem. Scott [四] further developed this method by creating arbitrary generalised viewpoints in an optimal scanning zone. Furthermore, he systematically analysed the required sensor performance model, included rotations about the sensor's optical axis into the view planning process and evaluated sensor pose uncertainty effects and countermeasures. Roberts et al. [日] maximise object visibility by searching for maximally connected subgraphs in a graph representation, where nodes representing object faces are connected, if they are visible from

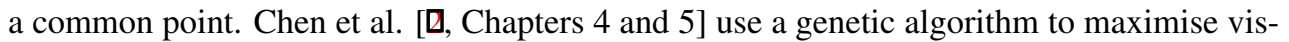
ibility using viewpoints on a sphere, while satisfying important constraints (e.g. resolution, viewing angle, reachability).

The extension of the statistical $E$-criterion to NBV planning was recently introduced by Trummer et al. []]. However, they perform an online path planning for a 3D reconstruction approach without active illumination, neglecting visibility and positioning constraints. Furthermore, their current approach explicitly tries to reduce the single greatest uncertainty of the current object model.

Munkelt et al. [G] proposed a benchmark method and test object for view planning. Using the benchmark procedures, one can quantitatively compare the results of different view planning approaches in terms of coverage, reconstruction error, and average point distance.

\section{Multi-View Planning}

Contrary to NBV planning, MVP requires a model of the object to be scanned. But the added complexity of providing a suitable model - either through a coarse scan using the same sensor [四], a CAD model or time-of-flight online 3D scan [] - enables the generation of more efficient view plans. Primary application areas are e.g. high fidelity scanning of complex objects and quality inspection of multiple, similar objects. Simulated and experimental results underline, that our method's overhead in computation time and model creation effort can be justified by the resulting higher coverage and accuracy, or shorter view plans, respectively.

In contrast to the majority of planning approaches, we model viewpoints $v$ as generalised viewpoints. Consequently, $v$ consists of sensor pose and a set of controllable sensor parameters $[\square, \boldsymbol{\square}]$. This also implies, that viewpoints $v$ are not restricted to e.g. a view sphere.

\subsection{Enhanced Coverage Using Adaptive Viewpoint Generation}

Our method's basic principle follows Scott's measurability matrix approach (3M) [四]. This involves generating "ideal candidate generalized viewpoints" (based on object and sensor 
model) and calculation of a binary matrix describing the measurability of each model point or triangle from each viewpoint candidate. This representation allows the determination of a minimal set of viewpoints (view set) scanning the whole models surface by mapping the problem to the one of minimal set cover (MSC, see for instance [ $\mathrm{Q}$, pp. $1033-1038$ ]). Finally, a view plan is derived from ordering the view set by e.g. previously unknown area scanned. For further details please refer to [ㅁ]].

For complex objects, analysis of the $3 \mathrm{M}$ viewpoint candidate creation scheme reveals some limitations. Candidates generated for surfaces in concavities may not be able to scan the target surface they originally were created for. Furthermore, optimal, yet unreachable (due to CS constraints) candidates may be created. While the $3 \mathrm{M}$ method handles object self occlusion and sensor positioning constraints in practice reasonably well, we improved the planners performance for complex measurement objects by an adaptive viewpoint generation.

Self Occlusion The original approach created ideal viewpoints for a model triangle $s$ by dilation along the surface normal $\mathbf{n}$ by the optimum scanning range $R_{o}$. This fails for many technical measurement objects containing structures such as notches. Often unfeasible viewpoints due to self occlusion by the object are produced. If we detect such occlusions during viewpoint creation, we search for optimal feasible positions $v^{\prime}$.

Configuration Space The original approach handled restricted CS by viewpoint filtering. As Scott mentioned, the advantages of ideal viewpoint creation are negated by excessive mobility constraints. However, practical inspection systems for large objects typically have significant mobility constraints. If we detect viewpoint candidates outside the CS, we also search for optimal feasible positions $v^{\prime}$.

Adaptive Viewpoint Generation In searching a feasible solution $v^{\prime}$ for an unfeasible viewpoint $v$, we want an alternative viewing direction with small angular error compared to $v$. Optimal alternative viewpoint candidates for a model triangle $s$ lie on a half sphere around $s$ with radius $R_{O}$ in surface normal direction. On this sphere, candidates on the same longitude share the same angular error. Longitudinal equiangular spaced candidates on a descending spiral around this sphere, starting at the pole, can be generated using a loxodrome (or rhumb line, see [四). If such an alternative passes the self occlusion and CS tests, a feasible solution is found. Otherwise, no viewpoint for $s$ is created. Due to incomplete sampling, $s$ may still be visible from other viewpoints (this is the standard situation for unfeasible viewpoints in the original approach).

\subsection{Accuracy Optimisation Using an Extended $E$-Criterion}

Most view planning approaches aim for complete acquisition of the measurement object. If quality is a concern, they aim for orthogonal surface scanning. An object's surface $s$ is assumed to be scannable, if a sensor pose exists, where $s$ can be scanned in compliance to the specification. Popular example is grazing angle threshold, ensuring that $s$ is scanned under a smaller angle between surface normal and viewing direction than e.g. $70^{\circ}$. Other tests may check sampling density, contrast, and other. Once a surface is (even poorly) scanned, further scanning quality improvement is often not aimed for (except e.g. [四]).

For high fidelity scanning we propose to include either an average or maximal measurement uncertainty requirement into MVP. We therefore expanded the scope of Sec. 3.1's view planning system beyond coverage, to model accuracy as well. By directly modelling 
uncertainty during view planning, a more accurate statement about the expected surface's scanning result than "scannable, exceeding minimal scanning requirements" can be made.

\subsubsection{Directional Uncertainty Estimation}

In order to extend the offline, MVP approach, the planner must be able to predict the measurement uncertainty. This also holds true for a posteriori evaluation of scans from reconstruction methods without dense explicit point correspondences between different views. Therefore we a priori calibrated a model of the sensor's measurement characteristic.

To this end, the sensor was positioned orthogonally in front of a plane at the distance of the optimal scanning range $R_{o}$. While enforcing identical measurement conditions, multiple scans are taken. Using identical object points along the sensor's boresight, the sensor's characteristic covariance matrix $\Sigma_{c}$ in standard position can be calculated.

Assuming normal distribution, $\Sigma_{c}$ defines an equiprobable curve of measurement uncertainty. Using eigen decomposition of $\Sigma_{c}$, we obtain eigenvalues $\lambda_{c}^{(1)} \geq \lambda_{c}^{(2)} \geq \lambda_{c}^{(3)}$ and corresponding perpendicular eigenvectors $\xi_{c}^{(1)}, \xi_{c}^{(2)}$ and $\xi_{c}^{(3)}$, which can be used to visualise $\Sigma_{c}$ as an ellipsoid. In accordance with the known $E$-criterion from statistics [छ], Trummer [ㅁ] formulated an extended $E$-criterion as sensing perpendicular to $\xi_{c}^{(1)}$, the direction of the largest uncertainty.

In order to apply this idea to offline MVP, it is necessary to solve two problems. Given a surface triangle $s$, its normal $\mathbf{n}$ and an arbitrary viewing direction $v_{i}$, we first must calculate the corresponding covariance matrix $\Sigma_{i}$ for scanning $s$ from viewpoint $v_{i}$. Second, covariance matrices from different scans of $s$ have to be combined to yield $\bar{\Sigma}_{n}$, the joined covariance matrix for surface $s$ after $n$ scans.

Calculating $\Sigma_{i}$ First, using rotation matrix $\mathbf{S}_{\mathbf{v}_{i}}$, we rotate $\xi_{c}^{(1)}, \xi_{c}^{(2)}$ and $\xi_{c}^{(3)}$, so that $\xi_{c}^{(1)}$ aligns with $v_{i}$ :

$$
\mathbf{Q}=\xi_{i}=\mathbf{R}_{\mathbf{v}_{i}} \mathbf{S}_{\mathbf{v}_{i}} \xi_{c},
$$

where $\mathbf{R}_{\mathbf{v}_{i}}$ rotates $\xi_{c}$ according to $\phi_{i}$, the sensor's roll angle around viewpoint $v_{i}$ 's boresight. Utilising the covariance matrix's symmetry and positive semi-definite properties, we construct $\Sigma_{i}$ by Eq. 2, where $\Lambda$ is the diagonal matrix of eigenvalues $\lambda_{c}$ :

$$
\Sigma_{i}=\mathbf{Q} \Lambda \mathbf{Q}^{\mathrm{T}} .
$$

Calculating $\bar{\Sigma}_{n} \quad$ For one model surface triangle $s$ we now have $i$ predicted covariance matrices $\Sigma_{i}$ from different viewpoints $v_{i}$. Since all observations of $s$ are modelled to share the same mean, we can use the average of the $\Sigma_{i}$ to obtain the cumulative covariance matrix $\bar{\Sigma}_{n}$ :

$$
\bar{\Sigma}_{n}=\frac{1}{n} \sum_{i=1}^{n} \Sigma_{i}
$$

In analogy to the standard error of the mean, defined as $S E=\sigma_{n} / \sqrt{n}$, we now define $S E_{\bar{\Sigma}_{n}}$ to both a priori evaluate the predicted measurement uncertainty of a candidate view set, as well as a posteriori evaluate the computed view set:

$$
S E_{\bar{\Sigma}_{n}}=\frac{\sigma_{n}}{\sqrt{n}} \equiv \frac{\sqrt{\lambda_{n}^{(1)}}}{\sqrt{n}} .
$$




\subsubsection{Extended $E$-Criterion}

For scans from arbitrary viewpoints $v_{i}$ measurement uncertainty rises, if the ideal scanning conditions during determination of $\Sigma_{c}$ are not met. Therefore we have to adapt $\Sigma_{i}$ accordingly. We chose to focus on incidence angle, focal depth and sampling density. For typical cooperative surfaces with lambertian reflectance, fringe contrast correlates strongly with incidence angle, so we did not consider it separately. Combined with Scott's approach, we thereby take 8 of 9 sensing constraints from [Q, chapter 4] into account. Furthermore, we calculate optimal viewpoint candidates $v_{i}^{n}$, depending on current scanning progress after $n$ views, the triangles surface normal $\mathbf{n}$, and the sensor's configuration space.

Quantitative Uncertainty Estimation In Sec. 3.2.1 we estimated the uncertainty's direction. To adapt to different levels of uncertainty arising from arbitrary measurement situations, one can scale the eigenvalues $\lambda_{c}$ accordingly. With incidence angle being the most prominent quality criterion $[\mathbf{Q}, \mathbf{\square}, \mathbf{Q}, \mathbf{⿴}]$ ], we divide $\lambda_{c}$ by $f_{i a}\left(\mathbf{n}, v_{i}\right)$, the minimum of dot products between the ray from surface $s$ to the sensor's emitting and receiving sub components (like projector or cameras) at viewpoint $v_{i}$, and the triangle's surface normal $\mathbf{n}$. For focal depth, we divide $\lambda_{c}$ by the weighted difference $d_{f d}$ between the distance $d_{s, v_{i}}$ from $s$ to viewpoint $v_{i}$ and the optimal scanning range $R_{o}$. The weighting function $f_{f d}\left(d_{f d}\right)$ is a sensor specific, experimentally parameterised bell-shaped function, which is 1 , if $d_{f d}=0$, and approximately 0.6 at the near focal distance. Finally, sampling density can be modelled as linearly decreasing with distance $d_{s, v_{i}}$. Thus we scale $\lambda_{c}$ linearly with the associated weighting function $f_{s d}\left(d_{s, v_{i}}\right)$ (slope sensor specific). In summary, $\lambda_{i}$ can be calculated as follows:

$$
\lambda_{i}=\frac{f_{s d}\left(d_{s, v_{i}}\right)}{f_{i a}\left(\mathbf{n}, v_{i}\right) \cdot f_{f d}\left(d_{f d}\right)} \lambda_{c} .
$$

We use $\lambda_{i}$ in Equation (2) to calculate the adapted covariance matrix $\Sigma_{i}$. Using Eq. (3), we can calculate $\bar{\Sigma}_{n}$, which in turn yields $\mathbf{v}_{1}=\xi_{n}^{(1)}$, the direction of the largest uncertainty.

E-Criterion Optimal Viewpoint Generation According to Trummer et al. [ $\square$ ], the optimal viewpoint for a given covariance estimation $\bar{\Sigma}_{n}$ is perpendicular to $\mathbf{v}_{1}$. This notion defines an optimal view plane $\pi_{s}(\mathbf{X}): \mathbf{v}_{1}^{\mathrm{T}}(\mathbf{X}-s)$ through e.g. the centre of gravity of triangle $s$, in which $E$-optimal viewpoints reside. The viewpoint with optimal viewing direction $\mathbf{0}_{E}$ regarding both $E$-criterion and incidence angle is the one with minimal angle to the triangle's surface normal $\mathbf{n}$ :

$$
\mathbf{o}_{E}=\frac{\mathbf{o}_{E}^{\prime}}{\left|\mathbf{o}_{E}^{\prime}\right|}, \text { with } \mathbf{o}_{E}^{\prime}=\mathbf{n}-\frac{\mathbf{n}^{\mathrm{T}} \mathbf{v}_{1}}{\mathbf{v}_{1}^{\mathrm{T}} \mathbf{v}_{1}} \mathbf{v}_{1} .
$$

However, the more similar $\lambda_{n}^{(1)}$ and $\lambda_{n}^{(2)}$ are (the error ellipsoid exhibits increasingly spheroid properties), the less descriptive is the $E$-optimal viewing direction $\mathbf{o}_{E}$, and the more important is the surface normal $\mathbf{n}$. To this end we calculate optimal viewing direction $\mathbf{o}$ as a weighted linear combination:

$$
\mathbf{0}=-\left(w_{n e} \mathbf{n}+\left(1-w_{n e}\right) \mathbf{o}_{E}\right),
$$

with $w_{n e}=\sqrt{\lambda_{n}^{(2)} / \lambda_{n}^{(1)}}$ the weight with regard to the ellipsoid's eccentricity. The resulting optimal viewpoint candidate $v$ for surface triangle $s$ is then computed by dilation along $\mathbf{o}$ by the optimum scanning range $R_{o}$. If unfeasible, $v^{\prime}$ is created according to Sec. 3.1. 

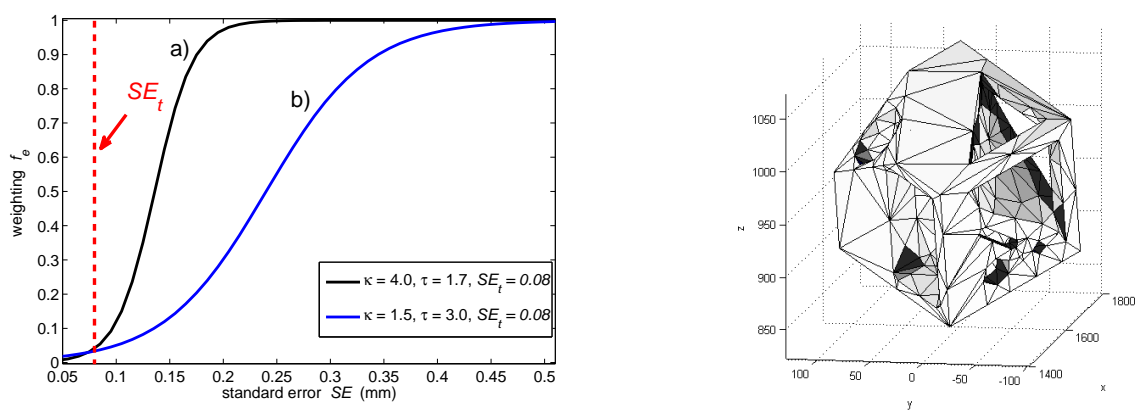

Figure 1: Left: Weighting of standard error $S E_{\bar{\Sigma}_{n}}$ for balancing between (a) accuracy and (b) completeness. Right: Visualization of the NBV test object's model evaluation after 3 views (lower uncertainty depicted by brighter shading).

\subsubsection{Iterative View Plan Calculation using the Extended $E$-Criterion}

To summarise, given an object's model and uncertainty information, we can calculate optimal viewpoints $v$ for surface patches $s$. If a scan of $s$ is performed from its derived $v$, the largest uncertainty component associated with $s$ is minimised. The object model is not limited to an appropriately processed CAD model, it can be as well a rough explorative scan or even a partial object model of the scanning progress so far. However, if a CAD model is available, multi-view planning can maximise completeness while limiting maximum measurement uncertainty to a certain level. In NBV approaches, exploring the scene generally involves suboptimal surface scans.

For model-based multi-view scanning, we start by calculating the initial viewpoints for all model triangles. Then we perform the measurability matrix calculations as in Scott's approach. Subsequently we calculate for each viewpoint and for each model triangle it's estimated cumulative covariance matrix $\bar{\Sigma}_{n}$ (Eq. 3) and corresponding standard error $S E_{\bar{\Sigma}_{n}}$ (Eq. 4). To choose the next best view, we select the view candidate, which decreases the current medium measurement uncertainty (over all triangles) the most. However, to account for different optimisation goals, we balance between coverage and accuracy by weighting $S E_{\bar{\Sigma}_{n}}$ according to the parameterised logistic function $f_{e}$ :

$$
f_{e}\left(S E_{\bar{\Sigma}_{n}}\right)=\frac{1}{1+\exp \left(-14 \kappa\left(S E_{\bar{\Sigma}_{n}}-\tau \cdot S E_{t}\right)\right)},
$$

with $\kappa$ the steepness (or accentuation) and $\tau$ the offset (or compliance) with respect to the target standard error $S E_{t}$ (see Figure 1 (left)). If triangle $s$ ' uncertainty is high, the gain of scanning $s$ is asymptotically one. The better $s$ is already scanned, the less is gained from rescanning $s$ to reduce its measurement uncertainty. By choosing a higher steepness, one accentuates accuracy in favour of completeness. By decreasing the offset, compliance to the chosen $S E_{t}$ is more strictly enforced.

The termination criterion (e.g. number of views or medium standard error) is tested after viewpoint selection. If more views are needed, we continue by updating the current cumulative covariance matrix for all triangles seen by the chosen viewpoint. Next, optimal viewpoints with respect to the current planning state are calculated according to Eq. (7). We then repeat the whole process by starting from the measurability matrix calculation above. 


\section{Experimental Results}

To examine our approach to view planning, we compared different planning strategies using the NBV benchmark from Munkelt et al. [छ]. It evaluates the planner's performance in reconstructing a complex measurement object (see Fig. 1 (right)) comprising four details. They challenge view planners with different difficulties, like self occlusion and challenging viewability. We analysed view count, coverage (the percentage of scanned compared to the scannable object surface) and reconstruction error (hausdorff distance between reconstruction and model) in accordance with the benchmark.

We performed the validation with a high resolution fringe projection system. It's sensor is composed of two cameras (triangulation angle approx. $20^{\circ}$ ) centred around a digital projector. The optimal scanning range $R_{o}$ is $750 \mathrm{~mm}$ and the depth of focus $\pm 100 \mathrm{~mm}$. The calibration procedure from Sec. 3.2.1 yielded a native point distance of approx. $0.150 \mathrm{~mm}$. Furthermore, the error ellipsoid's principal axis corresponds to the sensor's boresight, with lengths of $\sqrt{\lambda_{c}^{(1)}}=0.045 \mathrm{~mm}$ and $\sqrt{\lambda_{c}^{(2)}}=0.008 \mathrm{~mm}$. The cut off / break down angle was configured at $70^{\circ}$. For an abstract understanding of the sensor's positioning system's CS, imagine a spherical shell segment of valid sensor poses. The middle sphere surface at $R_{o}$ would cover approx. $33 \%$ of the area of a half sphere. The sensor is moved using a 6 DOF robot. Candidate viewpoints are not restricted to lie on a view sphere around the object.

The rough model used for view planning consisted of 434 triangles. Following Scott's decoupled algorithm for viewpoint generation, four rotations around the sensor's boresight in steps of $45^{\circ}$ are performed. The current planners prototypical implementation is done in MATLAB. Favouring flexibility over performance, the runtime could be reasonably shortened using optimisation and parallelisation. Using a Core 2 Duo @ $2.4 \mathrm{GHz}$, the adaptive viewpoint planner from Sec. 3.1 generates a view plan after approx. 23 min, the $E$-criterion based planner from Sec. 3.2 after approx. $101 \mathrm{~min}$. View planning for all approaches was performed without pose uncertainty modelling.

\subsection{Coverage evaluation}

Table 1 summarises the results for Sec. 3.1. For brevity, we compared coverage according to the NBV benchmark just for 3, 4, 6, 8 and the terminating number of views. Our "adaptive" approach is contrasted with the original approach from Scott ("baseline"). For both, the underlying MSC problem was solved optimally (using binary integer programming). In contrast, "expert" is a human planned view sequence. The total view plan length of the adaptive approach is 14 , compared to baseline and expert with 16 . After 8 views the adaptive view planning strategy reaches similar or better coverage in all categories.

We investigated, why predicted coverage differed from actually realised coverage. As it turns out, pose errors and inaccuracies in CS filtering led to discrepancies between planned surface area acquisition and actually scanned surface area. For further experiments we must therefore incorporate basic pose error compensation (as in [四, 四]).

We now compare two view plans created with different parameterizations for the weighting function $f_{e}$ of the approach from Sec. 3.2. While " $E$-crit 1 " $\left(\kappa=1.5 ; \tau=3.0 ; S E_{t}=0.08\right)$ balances coverage and accuracy, " $E$-crit 2 " ( $\left.\left[\kappa=4.0 ; \tau=1.7 ; S E_{t}=0.08\right]\right)$ favours accuracy over coverage. The planning cycle terminated after 8 views. Figure 2 shows their predicted coverage and standard error in addition to the results for the baseline and adaptive approach. Both approaches reach better coverage levels compared to "baseline" and "expert". 


\begin{tabular}{c|c||c||c|c|c|c|c|}
\multirow{2}{*}{$\begin{array}{c}\text { object } \\
\text { detail }\end{array}$} & \multicolumn{1}{c}{ 3D errors $[\mu \mathrm{m}]$} & \multicolumn{5}{c|}{ coverage $(\%)$} \\
\cline { 5 - 7 } (pethod & 8 views & 3 & 4 & 6 & 8 & total \\
\hline whole & baseline & $24 / \mathbf{5 2} / 96$ & 70.9 & 79.8 & 82.4 & 83.7 & 85.6 \\
\hline \hline predicted) & adaptive & $28 / 60 / 108$ & $\mathbf{7 7 . 0}$ & $\mathbf{8 1 . 5}$ & $\mathbf{8 5 . 6}$ & $\mathbf{8 7 . 8}$ & $\mathbf{9 0 . 3}$ \\
\hline whole & baseline & $9 / 21 / 42$ & 68.5 & 73.0 & 76.6 & 79.5 & 82.4 \\
(real) & adaptive & $9 / \mathbf{1 8} / 35$ & $\mathbf{7 4 . 2}$ & $\mathbf{7 9 . 3}$ & $\mathbf{8 4 . 2}$ & $\mathbf{8 6 . 1}$ & $\mathbf{8 7 . 6}$ \\
& expert & $9 / \mathbf{1 8} / 35$ & 70.0 & 76.3 & 80.2 & 80.8 & 84.7 \\
\hline notch & baseline & $6 / 12 / 26$ & 55.3 & $\mathbf{7 0 . 6}$ & 72.5 & $\mathbf{7 6 . 8}$ & 78.2 \\
& adaptive & $6 / 15 / 32$ & $\mathbf{6 3 . 6}$ & 63.6 & $\mathbf{7 6 . 8}$ & $\mathbf{7 6 . 8}$ & $\mathbf{8 3 . 0}$ \\
& expert & $5 / \mathbf{1 1} / 25$ & 38.6 & 68.0 & 69.6 & 71.5 & 74.9 \\
\hline neg. sphere & baseline & $14 / 27 / 41$ & 32.4 & 32.4 & 59.3 & 59.8 & 64.4 \\
& adaptive & $9 / \mathbf{1 9} / 33$ & $\mathbf{6 3 . 9}$ & $\mathbf{6 4 . 1}$ & $\mathbf{7 7 . 9}$ & $\mathbf{7 8 . 0}$ & $\mathbf{8 3 . 4}$ \\
& expert & $27 / 43 / 61$ & 47.2 & 47.4 & 50.5 & 50.6 & 54.5 \\
\hline \multirow{2}{*}{ sinusoidal } & baseline & $30 / 43 / 54$ & $\mathbf{6 7 . 2}$ & 67.2 & 69.8 & 80.8 & 88.7 \\
& adaptive & $21 / 40 / 58$ & 61.9 & $\mathbf{8 3 . 4}$ & $\mathbf{8 7 . 8}$ & $\mathbf{9 1 . 1}$ & 91.2 \\
& expert & $13 / \mathbf{2 4} / 35$ & 61.4 & 61.4 & 79.5 & 79.5 & $\mathbf{9 4 . 3}$ \\
\hline tripod & baseline & $6 / \mathbf{1 4} / 27$ & 50.1 & 59.8 & 63.6 & 64.5 & 64.5 \\
& adaptive & $6 / \mathbf{1 4} / 34$ & $\mathbf{6 9 . 7}$ & $\mathbf{7 1 . 1}$ & 72.6 & $\mathbf{7 6 . 9}$ & $\mathbf{7 7 . 2}$ \\
& expert & $6 / 15 / 28$ & $\mathbf{6 9 . 7}$ & 70.6 & $\mathbf{7 2 . 9}$ & 73.3 & 74.8
\end{tabular}

Table 1: Coverage results using the optimal MSC solution (best value bold, total views: baseline 16 , adaptive 14 , expert 16 ). 3D errors are given for the $1^{\text {st }}, 2^{\text {nd }}$ and $3^{\text {rd }}$ quartile.
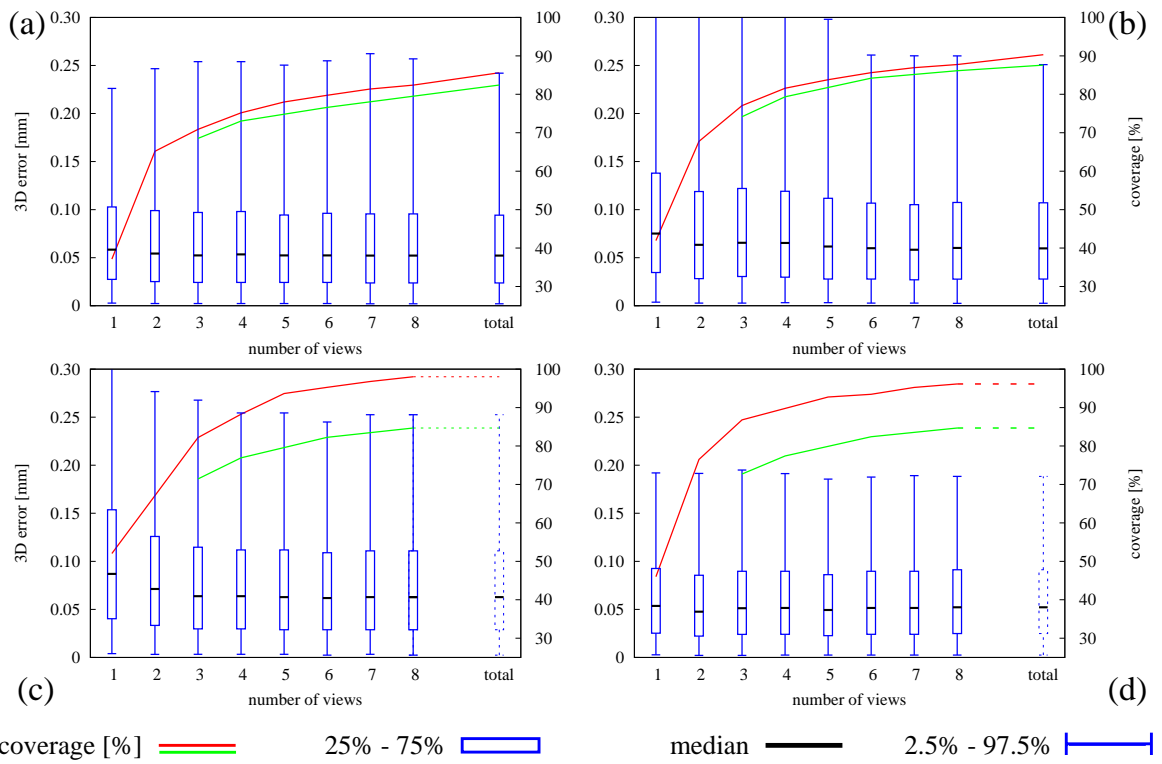

Figure 2: Comparison of predicted (red) and realised (green) coverage, and accuracy for the whole NBV test object: (a) baseline approach; (b) adaptive viewpoint generation; (c) $E$-criterion with $\left[\kappa=1.5 ; \tau=3.0 ; S E_{t}=0.08\right]$; (d) $E$-criterion with $\left[\kappa=4.0 ; \tau=1.7 ; S E_{t}=0.08\right]$. 


\begin{tabular}{|c|c|c|c|c|c|c|}
\hline \multirow{2}{*}{$\begin{array}{l}\text { object } \\
\text { detail }\end{array}$} & \multirow[b]{2}{*}{ method } & \multirow{2}{*}{$\begin{array}{c}3 \mathrm{D} \text { errors }[\mu \mathrm{m}] \\
8 \text { views }\end{array}$} & \multicolumn{4}{|c|}{ coverage $(\%)$} \\
\hline & & & 3 & 4 & 6 & 8 \\
\hline \multirow{2}{*}{$\begin{array}{c}\text { whole } \\
\text { (predicted) }\end{array}$} & $E$-crit ${ }_{1}$ & $29 / 63 / 111$ & 82.2 & 88.3 & 95.3 & 98.0 \\
\hline & $E$-crit 2 & 25 / 52 / 91 & 86.8 & 89.8 & 93.5 & 96.2 \\
\hline \multirow[t]{2}{*}{ whole } & $E$-crit ${ }_{1}$ & $10 / 21 / 41$ & 71.4 & 76.9 & 82.3 & 84.7 \\
\hline & $E$-crit 2 & $6 / 16 / 31$ & 72.7 & 77.4 & 82.4 & 84.7 \\
\hline \multirow[t]{2}{*}{ notch } & $E$-crit ${ }_{1}$ & $6 / 13 / 26$ & 60.4 & 71.7 & 71.8 & 77.1 \\
\hline & $E$-crit 2 & $7 / 15 / 33$ & 61.8 & 61.8 & 78.1 & 81.0 \\
\hline \multirow[t]{2}{*}{ neg. sphere } & $E$-crit ${ }_{1}$ & $20 / 43 / 72$ & 65.6 & 66.0 & 66.1 & 66.5 \\
\hline & $E$-crit 2 & $12 / 25 / 39$ & 59.3 & 59.4 & 75.1 & 76.3 \\
\hline \multirow[t]{2}{*}{ sinusoidal } & $E$-crit ${ }_{1}$ & $16 / 29 / 40$ & 58.2 & 58.3 & 87.4 & 87.6 \\
\hline & $E$-crit 2 & 15 / 27 / 39 & 59.8 & 79.8 & 80.1 & 87.2 \\
\hline \multirow[t]{2}{*}{ tripod } & $E$-crit ${ }_{1}$ & $7 / 15 / 31$ & 57.4 & 71.8 & 72.2 & 78.0 \\
\hline & $E$-crit 2 & $6 / 12 / 30$ & 64.8 & 66.4 & 70.5 & 71.5 \\
\hline
\end{tabular}

Table 2: Coverage results using the greedy MSC solution (best value bold). 3D errors are given for the $1^{\text {st }}, 2^{\text {nd }}$ and $3^{\text {rd }}$ quartile.

Since the underlying MSC problem solving strategy is inherently an iterative greedy approach, Tab. 2 separately summarises the NBV benchmark coverage results. " $E$-crit 1 " can maximise coverage, while fulfilling the accuracy requirement. Overall, coverage is lower compared to our adaptive planning strategy, yet similar or better than the baseline approach, despite the less optimal, iterative MSC solution.

\subsection{Accuracy evaluation}

Figure 2 shows the predicted measurement uncertainty for four planning methods. The $E$ criterion based view planning clearly shows continually decreasing maximal, as well as median errors. While reaching similar coverage, both methods realise better accuracy (lower interquartile range and smaller maximum reconstruction errors). As expected, " $E$-crit 2 " exhibits better accuracy than " $E$-crit 1 ", at the expense of slower coverage growth.

Figure 3 shows reconstruction error evaluation according to the method described in [ब]. After 8 views, our " $E$-crit 1 " method achieves slightly better error values, compared to all other methods. The error levels for the sinusoidal face are generally higher than the other object details due to difficult accessibility with the scanner. Yet our $E$-criterion algorithm produces the lowest errors. For the "notch" and "tripod" details our maximum error is higher, while still maintaining similar median error levels. Between predicted and realised errors there is approximately a factor of 2 , which originates from activated post processing filters during scanning. These filters were disabled during calibration of sensor characteristics.

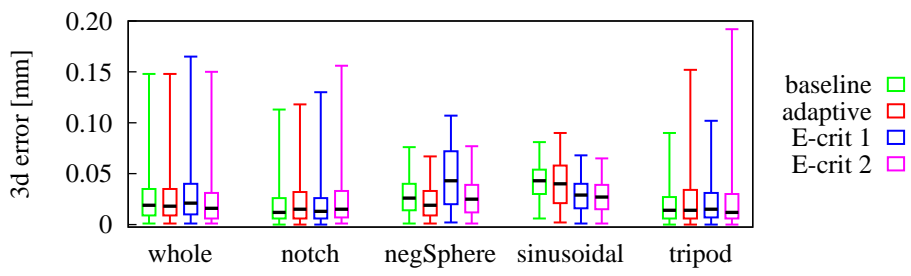

Figure 3: Realised reconstruction errors (after 8 views). 


\section{Conclusion}

In this paper we presented an offline multi-view planning approach for simultaneously optimising reconstruction completeness and accuracy of complex objects. We proposed a method for estimating uncertainty, which in turn allows to formulate the extended $E$-criterion for accuracy optimisation. We integrated the planning procedure into a minimal set cover based approach appropriate for high fidelity scanning. The method is suitable for robot mounted, fringe projection based stereo 3D scanning with notable configuration space constraints. Simulation and experimental evaluation show enhanced coverage, and partially improved accuracy. Future work will address the inclusion of pose error into the planning to enhance planning robustness.

\section{References}

[1] J. Alexander. Loxodromes: A rhumb way to go. Mathematics Magazine, 77(5):349356, December 2004.

[2] S.Y. Chen, Y.F. Li, J.W. Zhang, and W.L. Wang. Active Sensor Planning for Multiview Vision Tasks. Springer-Verlag, 2008.

[3] T. H. Cormen, C. E. Leiserson, R. L. Rivest, and C. Stein. Introduction to Algorithms, second edition. 2001. doi: 10.1.1.111.5804.

[4] N. A. Massios and R. B. Fisher. A Best Next View selection algorithm incorporating a quality criterion. In 9th BMVC, pages 780-789, September 1998.

[5] C. Munkelt, M. Trummer, J. Denzler, and S. Wenhardt. Benchmarking 3D reconstructions from next best view planning. In Proceedings of IAPR Conference on Machine Vision Applications, pages 552-555, May 2007.

[6] C. Munkelt, M. Trummer, P. Kühmstedt, G. Notni, and J. Denzler. View planning for 3D reconstruction using time-of-flight camera data. In Proceedings of 31st DAGM Syposium (LNCS 5748), pages 352-361. Springer-Verlag, September 2009.

[7] R. Pito. A Solution to the Next Best View Problem for Automated Surface Acquisition. IEEE Transactions on Pattern Analysis and Machine Intelligence, 21(10):1016-1030, October 1999.

[8] F. Pukelsheim. Optimal Design of Experiments. John Whiley \& Sons Inc., New York, 1993.

[9] D. R. Roberts and A. D. Marshall. Viewpoint Selection for Complete Surface Coverage of Three Dimensional Objects. In British Machine Vision Conference, pages 740-750, Department of Computer Science, Cardiff University, PO Box 916, Cardiff. CF2 3XF, U.K., 1998.

[10] W. R. Scott. Model-based view planning. Machine Vision and Applications, 20(1): 47-69, January 2009. doi: 10.1007/s00138-007-0110-2. 
[11] W. R. Scott, G. Roth, and J.-F. Rivest. View planning for automated three-dimensional object reconstruction and inspection. ACM Computing Surveys, 35(1):64-96, March 2003.

[12] G. H. Tarbox and S. N. Gottschlich. Planning for complete sensor coverage in inspection. Comput. Vis. Image Underst., 61(1):84-111, 1995. ISSN 1077-3142. doi: http://dx.doi.org/10.1006/cviu.1995.1007.

[13] M. Trummer, C. Munkelt, and J. Denzler. Online next-best-view planning for accuracy optimization using an extended e-criterion. Proceedings of IAPR 20th International Conference on Pattern Recognition (ICPR) (to appear), 2010. 